\title{
Evaluation of autonomic nervous system by heart rate variability and differential count of leukocytes in athletes
}

\author{
Nobuhiro Suetake $^{1,2 *}$, Yukiko Morita $^{2}$, Daichi Suzuki ${ }^{3}$, Keiko Lee ${ }^{1}$, Hiroyuki Kobayashi $^{1}$ \\ ${ }^{1}$ Department of Hospital Administration, Juntendo University, School of Medicine, Tokyo, Japan \\ ${ }^{2}$ Sakae Clinic, Aichi, Japan; *Corresponding Author: nobu666@d1.dion.ne.jp \\ ${ }^{3}$ Department of Sports Science, Juntendo University, Graduate School of Health and Sports Science, Chiba, Japan
}

Received 22 May 2010; revised 15 June 2010; accepted 2 July 2010.

\begin{abstract}
Top Japanese sprinters were evaluated for their physical condition, autonomic function, blood chemistry, differential leukocyte count and blood lactate level before and after short, maximal exercise to explore methods of quantifying their conditioning level. Statistical analysis of data obtained before and $10 \mathrm{~min}$ after exercise were used to estimate the athletes' autonomic capacity during recovery. Pre and post exercise variances in differential leukocyte count revealed strong correlations between neutrophil and sympathetic activity, and lymphocyte and parasympathetic activity. The results of the study demonstrated significant alterations in autonomic parameters and differential white blood cell count in response to maximal exercise.
\end{abstract}

Keywords: Heart Rate Variability; Conditioning; Differential Count of Leukocytes

\section{INTRODUCTION}

It is of paramount importance for high-performance athletes to consistently train in a manner that preserves their optimal conditioning level. Athletes regularly train at high intensity and volume for extended periods such that an imbalance between excessive workload and inadequate recovery renders them susceptible to mental and physical depletion. Therefore, athletes 'fitness level is an important factor in designing training programs and for preventing overtraining syndrome [1]. However, previous attempts to portray the blood lactate level and Profile of Mood State (POMS) as respective indicators of physical and mental fatigue have not been entirely conclusive. For this study, heart rate variability (HRV) analy- sis [2] has been selected to quantitatively evaluate autonomic capacity $[3,4]$ for its greater accuracy in estimating physical fitness than earlier methods [5]. Cardiac rhythm is modulated by the two limbs of the autonomic nervous system (ANS), the sympathetic and parasympathetic, which exert antagonistic effects. Sympathetic dominance occurs during stressful conditions such as nervousness and excitement, whereas a shift in favor of vagal modulation calms the heart rate. Accordingly, heart rate and RR interval fluctuation (HRV) permit analogical inference of autonomic influence as well as the effects of mental state and stress level on sympathetic and vagal outputs (HRV analysis) [6,7].

Abo has previously reported that leukocytes are under autonomic control [8]. More specifically, sympathetic and parasympathetic stimulations have been shown to activate adrenalin receptors on granulocytes, the most prolific leukocytes, and acetylcholine receptors on lymphocytes, respectively [9]. Leukocytes have two major biological defense activities. Granulocytes and macrophages incite phagocytic activities against foreign bacteria and materials, whereas lymphocytes respond to viral and abnormal protein infiltrations by mediating antigenantibody production and attacking cellular damage.

In this study we have focused on the proportion of neutrophil, the representative granulocyte, and lymphocyte subpopulations. In short, we have explored whether the alterations in autonomic parameters and granulocyte/lymphocyte ratio serve as novel markers of physical and mental exhaustion in sprinters after maximal high knee lifts, and also act as potential indicators of athletes' fitness level.

\section{MATERIALS AND METHODS}

\subsection{Subjects}

Five elite sprinters in their twenties (mean age $27 \pm 1.4$ ) 
with qualifications or titles in the Prefectural Track and Field Championships participated in the study (1 female and 4 male sprinters with personal bests ranking among the top 20 in 2009). Weekly training program for the athletes consisted of daily circuit and weight training for $>/=3 \mathrm{hr}$ /day for more than 5 days a week, punctuated with a day of rest and participation in competitive and time trials on Sundays. All participants routinely engaged in physical exercise for $>/=3 \mathrm{hr} /$ day for at least 5 times per week. Subjects were fully informed about the objectives, specific test procedures, and mental and physical burden of participating in the study. Test times were scheduled to avoid conflict with competitions and to prevent carryover effects on athletic performance. Eligible participants provided full consent before enrolling in the study. Subjects performed a light warm-up before the test exercise to assess their condition. All study participants were nonsmokers and had no history of hypertension, hyperlipidemia, cardiovascular disease, or diabetes. None of the athletes were taking cardiovascular medications and psychiatric agents such as sleeping pills and anxiolytic drugs. On the day of the test, subjects must have had a body temperature of $>/=37^{\circ} \mathrm{C}$, absence of significant malaise, $>/=6$ hours of sound sleep and abstinence from alcohol intake for at least $24 \mathrm{hr}$ prior to the test. Participants were instructed to complete voiding and avoid food and drink for more than $3 \mathrm{hr}$ before the scheduled test time. Pre-exercise measurements were taken in the sitting position after a 20-min rest. Subjects were monitored to pace their respiration at approximately 13 breaths/min.

\subsection{Method}

Heart Rate Variability (HRV) Analysis: By measuring heart rate and RR interval fluctuations (HRV), autonomic activity and the effects of mental balance and stress level on this capacity can be analogically inferred. RR interval is the distance between two consecutive $\mathrm{R}$ waves which are known as the largest spikes in EKG. Spectral transformation of this RR interval variability generates high frequency power called "HF" $(>0.15 \mathrm{~Hz})$ and low frequency power termed "LF" $(0.04 \sim 0.15 \mathrm{~Hz})$, where HF power is regarded as the primary estimate of cardiac vagal activity. HF power is also generally presumed to correspond to respiratory sinus arrhythmia and therefore approximates to the frequency of respiration. In contrast, LF power is surmised as mirroring the activities of the two autonomic nerves. For these reasons, the ratio of HF and LF is considered as the index of sympathovagal activity. The LF/HF ratio is typically associated with sympathetic activation and HF with vagal excitation. These frequency domain indices are obtained from power spectral analysis that has been calculated from the fast

\section{Fourier transform (FFT) [10].}

Primary outcome parameters for this study were frequency domain parameters such as very low frequency (VLF), high frequency (HF), low frequency (LF), LF/HF ratio, total power (TP). Additionally, blood chemistry (AST, ALT, LDH, CK, blood glucose, BUN); differential WBC count; and blood lactate levels were obtained before and $10 \mathrm{~min}$ after all-out high knee lifts. HF Norm (adjusted HF) and LF Norm (adjusted LF) were used in lieu of HF and LF for statistical analyses.

$$
\begin{aligned}
& \text { HF Norm }=\text { HF } /(\text { Total Power }- \text { VLF }) \times 100 \\
& \text { LF Norm }=\text { LF } /(\text { Total Power }- \text { VLF }) \times 100
\end{aligned}
$$

The purpose of adjusting the values was to minimize the impact of VLF alterations and to emphasize the separate fluctuations induced by parasympathetic and sympathetic activities. VLF has recently garnered attention as an important prognostic indicator of ischemic heart disease [11]. Although VLF is considered to reflect the very slow mechanistic activity of sympathetic control, the underlying physiological mechanisms remain unclear. Consequently, VLF measurements were omitted from this study and the adjusted values were used for statistical analysis.

Based on the recommendation by The European Society of Cardiology and the North American Society of Pacing and Electrophysiology, resting HRV was measured with the Heart Rhythm Scanner (HRV analysis system from Biocom, Inc.) equipped with software that performs algorithms of short-term HRV analysis. Measurements were taken from subjects in sitting in a quiet room before the exercise. Biocom HRM-02 Pulse Wave Sensor, the photoplethysmography (PPG) monitor used in the study, was clipped to the right earlobe to measure HRV for 5 minutes. The same procedure was repeated after exercise. The PPG monitor was placed above the converter (light $\rightarrow$ energy) for the light source and photocells and adjacent to both earlobes to direct the infrared beam towards the photocells. Since blood volume fluctuations within the vasculature of the earlobe correlate to beat-to-beat changes, the PPG was employed to capture the pulse waves to generate data on HRV. At a designated area of a temperature $\left(24^{\circ} \mathrm{C}\right)$ and humidity $(60 \%)$ controlled testing room, participants performed two sets of 1-min all-out high knee lifts interspersed with 1-min of passive recovery. Tests were conducted between 12:00 PM - 18:00 PM when HRV was least variable. In order to induce complete exhaustion, a supervising trainer provided verbal cues throughout the test period to monitor and ensure the pace of the exercise. Participants rested in standing during the 1-min interval and in supine for 10 minutes during post-exercise recovery. The same pre-exercise autonomic assessment and blood tests were repeated $10 \mathrm{~min}$ after the exercise to 
measure and quantitatively evaluate autonomic functions. Participants were instructed to maintain a consistent respiration rate for the autonomic assessment during recovery. Concomitantly with HRV analysis, routine blood tests for assessing conditioning level have been evaluated in this study, including: 1) blood chemistry aspartate amino transferase (AST), alanine amino transferase $(\mathrm{ALT})$, lactate dehydrogenase (LDH), creatine kinase (CK), blood urea nitrogen (BUN) to examine kidney functions and protein metabolism; 2) blood lactate concentration to assess glucose metabolism; and 3) differential WBC count to investigate recent appraisal of the correlation between sympathovagal activity and leukocyte subsets (neutrophils, lymphocytes, monocytes, eosinophils and basophils). Pre and post exercise blood samples were obtained from the same median cubital vein and sent to FALCO biosystems Ltd to be tested. Subjects were immediately asked about their fatigue rating after the exercise. All laboratory tests and physical measurements were taken while subjects sat calmly in the test room.

Blood chemistry, differential WBC count, blood lactate level: The following measurements were taken before and after the stress exercise: 1) blood glucose (BG) to observe energy expenditure during exercise; 2) aspartate amino transferase (AST), alanine amino transferase (AL$\mathrm{T}$ ), lactate dehydrogenase $(\mathrm{LDH})$, and creatine kinase (CK) to evaluate injury/inflammation of muscle tissues; 3) blood urea nitrogen (BUN) to assess alterations in ki- dney functions and protein metabolism; 4) blood lactate concentration to determine glucose metabolism; and 5) differential WBC count (neutrophils, lymphocytes) to assess autonomic capacity.

\subsection{Statistical Analysis}

T-test with the level of significance set at $\mathrm{P}<0.05$ was used to assess pre and post exercise variations. All statistical analyses were carried out with PASW Statistics 17.0 (SPSS Inc.) software.

\section{RESULTS}

\subsection{HRV Analysis (Figures 1 - 5)}

The autonomic parameters of all subjects, including total power (TP), very low frequency (VLF), high frequency (HF), low frequency (LF) and LF/HF ratio during rest were all within normal limits.

Figures 1-5 show the comparisons of mean TP, VLF, LF Norm, HF Norm and LF/HF ratio among the five sprinters.

Comparisons of mean TP, VLF, LF Norm, HF Norm, and $\mathrm{LF} / \mathrm{HF}$ ratio of the five sprinters before and $10 \mathrm{~min}-$ ute after maximal high knee lifts demonstrated a significant decrease in TP $(p<0.05)$, LF Norm $(p<0.01)$ and $\mathrm{LF} / \mathrm{HF}$ ratio $(\mathrm{p}<0.05)$, while a considerable increase in HF Norm $(p<0.05)$ was noted. VLF tended towards a decline despite statistical insignificance $(\mathrm{p}<0.1)$.

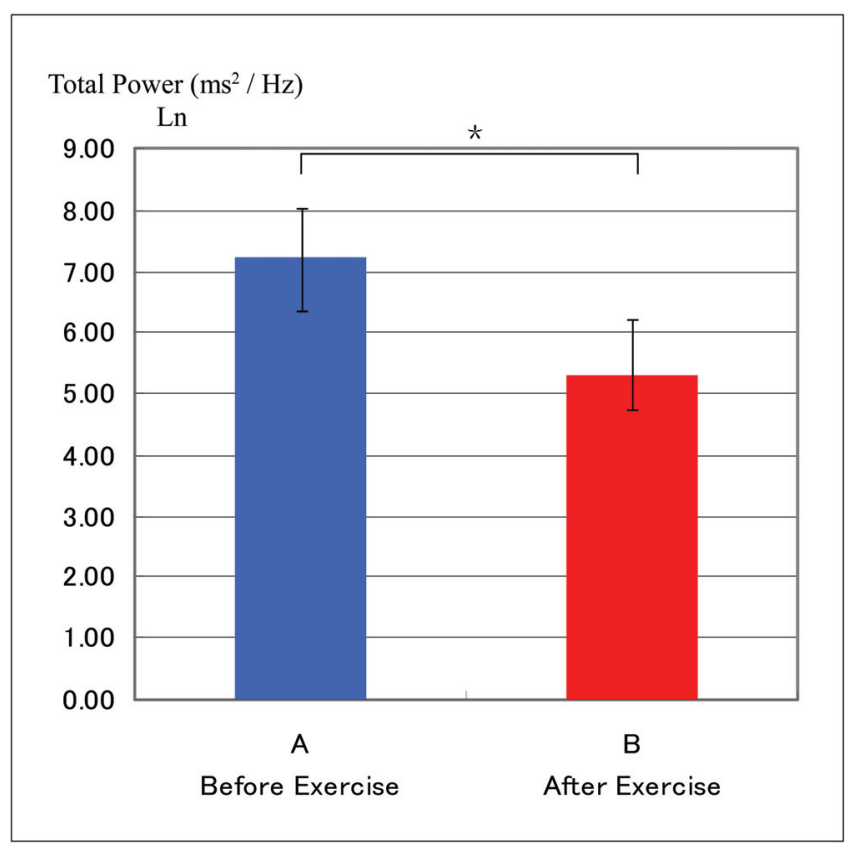

Figure 1. Comparison of mean total power among sprinters before and 10 minute after all-out high knee lifts. A significant decrease $(p<0.05)$ was observed after the exercise stress test. 


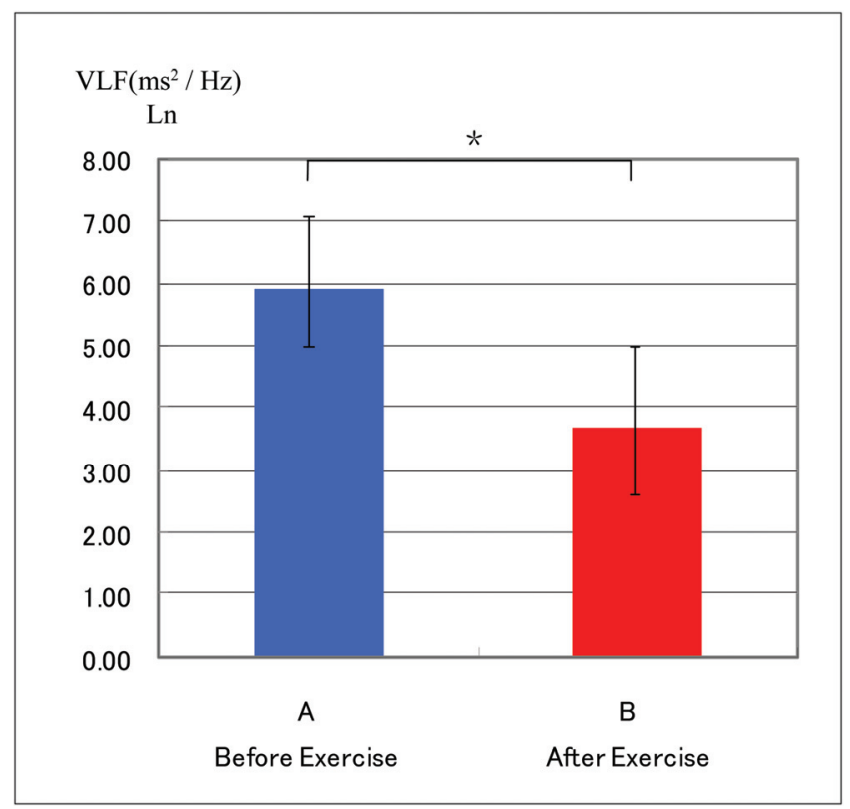

Figure 2. Comparison of mean VLF among sprinters before and 10 minute after all-out high knee lifts. A trend towards decline was noted after the exercise stress test despite statistical insignificance $(\mathrm{p}<0.1)$.

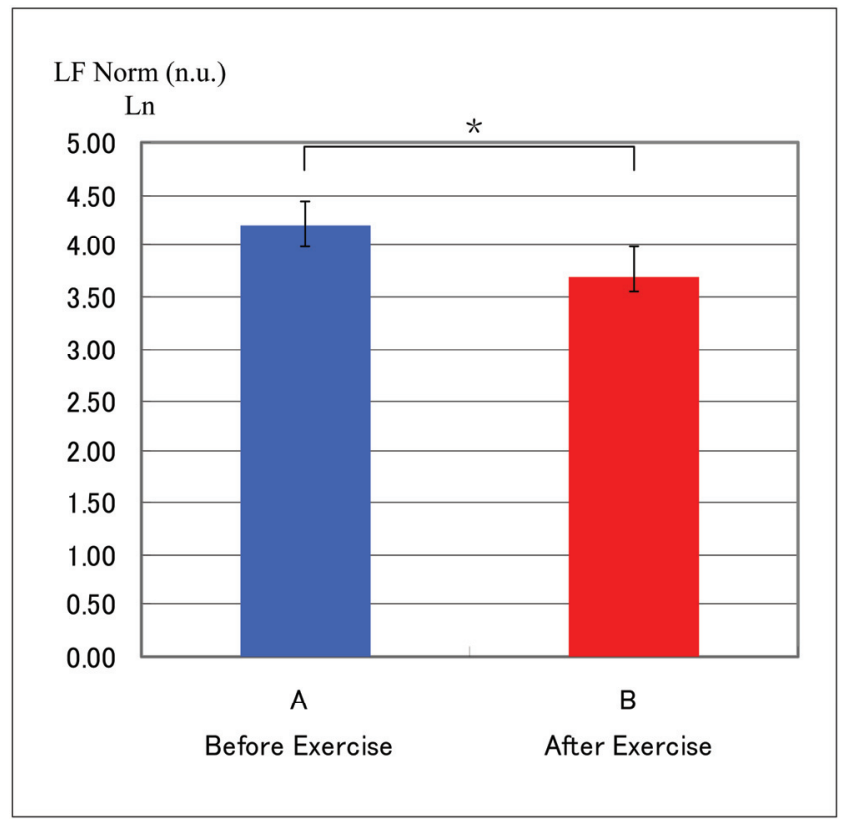

Figure 3. Comparison of mean LF Norm among sprinters before and 10 minute after all-out high knee lifts. A significant decrease $(p<0.01)$ was observed after the exercise stress test.

\subsection{Blood Chemistry, Blood Lactate Concentration}

Blood chemistry and blood lactate level were all within normal limits before and after exercise. All aspects of blood chemistry of pre and post exercise samples did not demonstrate significant changes, except for creatine kinase $(\mathrm{CK})$. Creatine kinase $(\mathrm{CK})$ and blood lactate levels increased significantly 10 minutes after short, maximal exercise $(\mathrm{p}<0.01)$. 


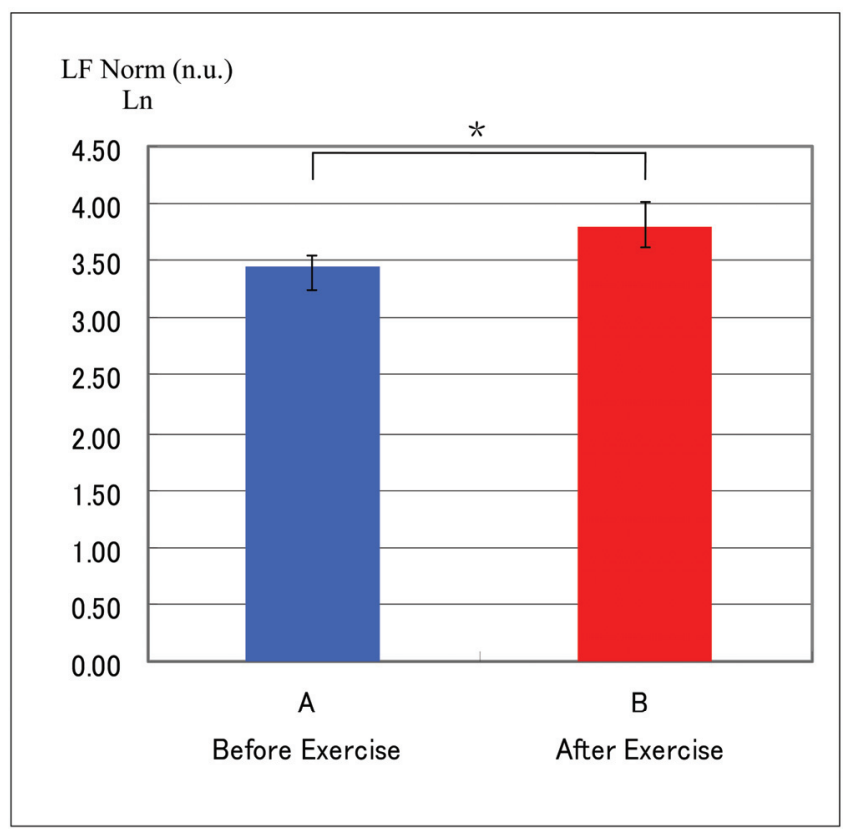

Figure 4. Comparison of mean HF Norm among sprinters before and 10 minute after all-out high knee lifts. A marked increase $(\mathrm{p}<$ $0.05)$ was observed after the exercise stress test.

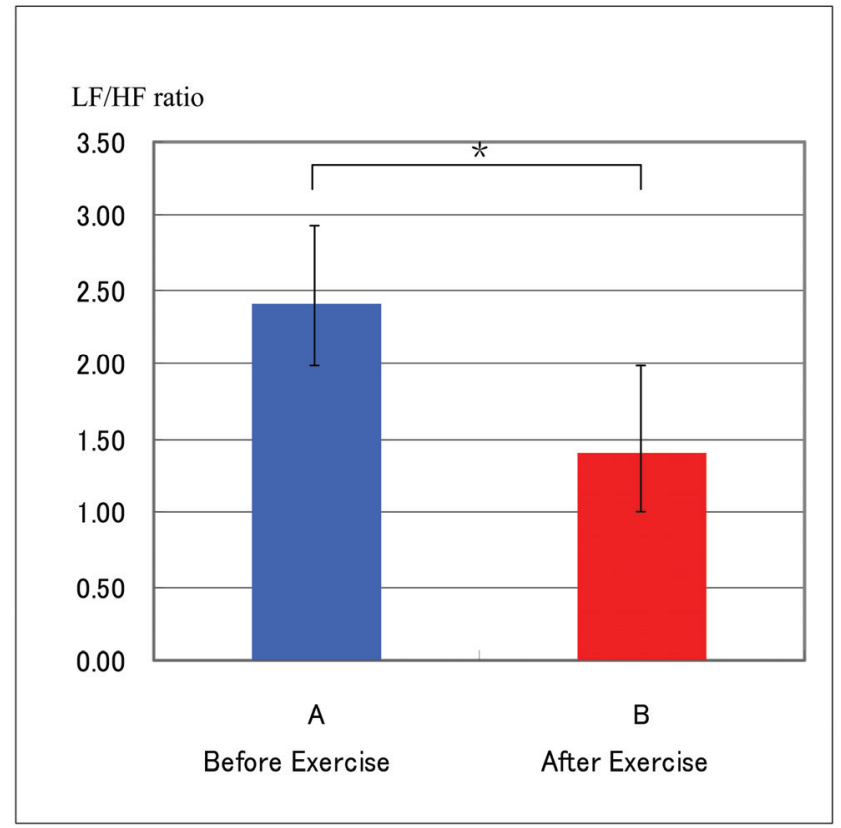

Figure 5. Comparison of mean $\mathrm{LF} / \mathrm{HF}$ ratio among sprinters before and 10 minute after all-out high knee lifts. A significant decrease $(p<0.05)$ was observed after the exercise stress test.

\subsection{Differential Leukocyte Count (Figures 6-7)}

Figures 6-7 illustrate the comparisons of mean neutrophil and lymphocyte ratios among the five sprinters be- fore and after all-out high knee lifts. The proportions of neutrophils and leukocytes in differential WBC count were all within normal limits. Neutrophils reduced significantly after exercise $(\mathrm{p}<0.01)$, while lymphocytes increased significantly after exercise $(\mathrm{p}<0.01)$. 


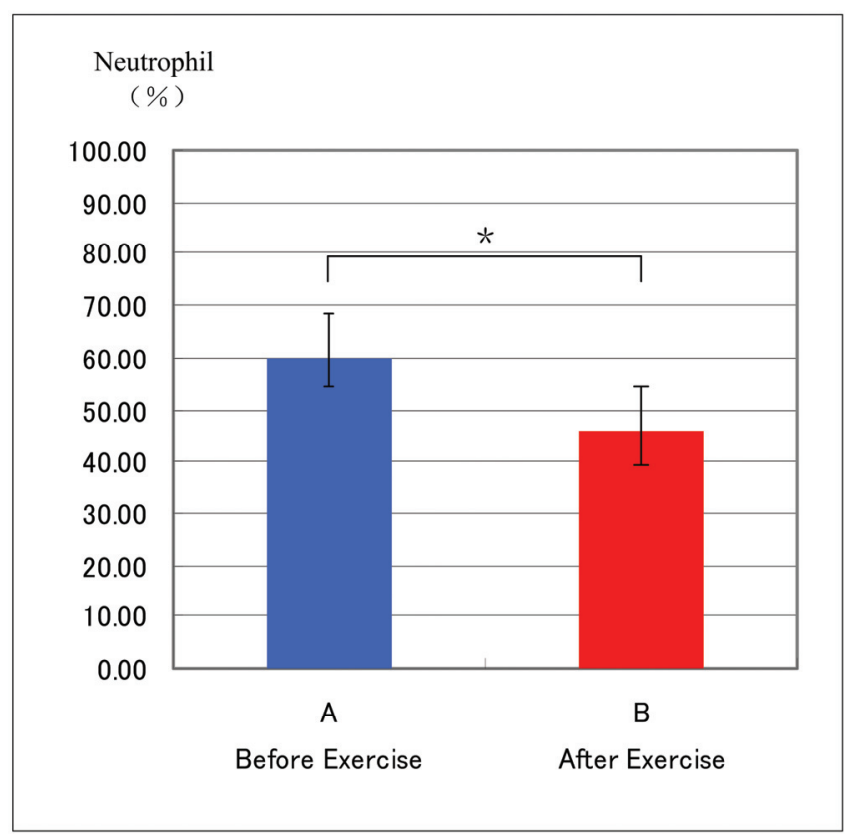

Figure 6. Comparison of mean neutrophil count among sprinters before and 10 minute after all-out high knee lifts. A significant decrease $(p<0.01)$ was observed after the exercise stress test.

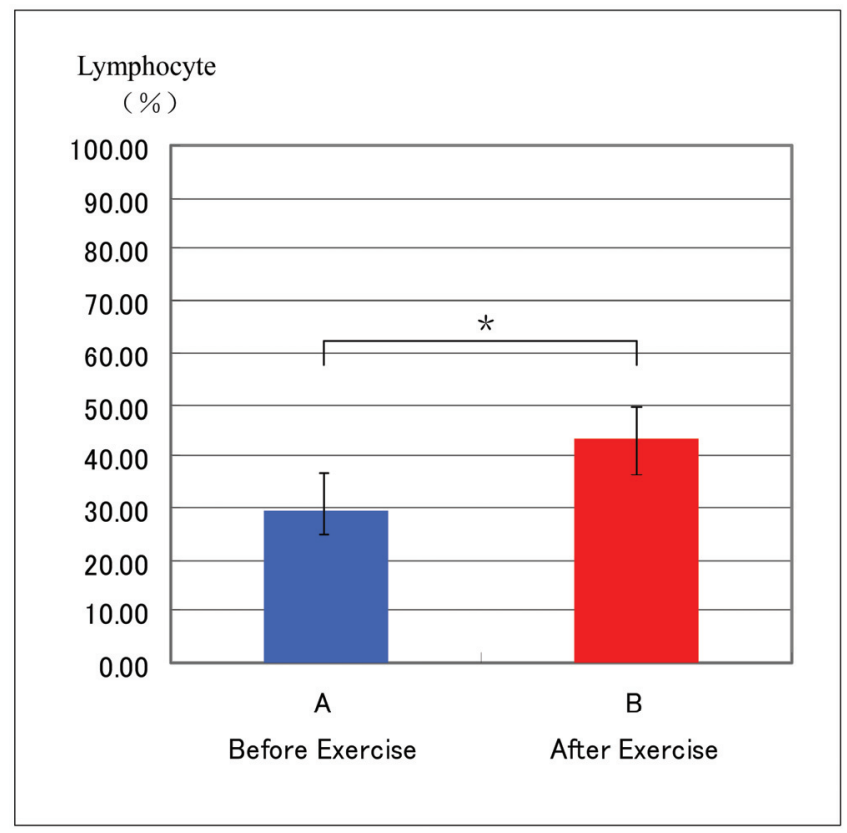

Figure 7. Comparison of mean leukocyte count among sprinters before and 10 minute after all-out high knee lifts. A significant increase $(p<0.01)$ was observed after the exercise stress test.

\section{DISCUSSION}

A battery of blood tests, psychological assessments and autonomic evaluations are routinely administered to athletes to objectively quantify their conditioning level. The importance of autonomic capacity in athletic performance has been elucidated, and reports on autonomic evaluation using HRV analysis in athletes across various disciplines are available [12]. Despite recent accounts underpinning the correlation between autonomic activity 
and white blood cell count, data on this relationship in the field of sports and training are practically nonexistent. Although leukocytosis is a well-established phenomenon following physical activity, and while reports on differential WBC alterations in response to exercise have been published [13], the relationship between pre and post differential leukocyte and autonomic activity remains equivocal. The present study focused on quantitative evaluation of pre and post exercise autonomic activities as well as differential leukocyte count in athletes.

Because the study targeted elite athletes who train regularly at high intensity and volume, all-out high knee lifts were selected as the exercise stress test to induce exhaustion. In the study, a prominent rise in peak lactate after maximal high knee lifts was noted which served as an objective marker of exhaustion in participants. Total power significantly decreased after exercise, but this was seen as a transient suppression of autonomic functions under substantial physical stress. Caution must be exercised when training athletes with reduced total power as this could indicate autonomic dystonia. The association between autonomic impairment and overtraining syndrome, a chronic state of fatigue, has been reported elsewhere. In the present study, significant post-exercise LF reduction was attributed to the inhibition of baroreceptor modulation in spite of sympathetic excitation after maximal exercise. In fact, LF appeared contingent not only on sympathetic tone but baroreceptor reflex as well.

In response to a sudden increase in heart rate after short maximal workload, parasympathetic tone is thought to amplify 10 minutes after exercise to counteract the sympathetic surge. On this basis, post-exercise measurements for this study were taken 10 minutes after brief maximal load to assess changes during recovery. Modulation of acutely augmented heart rate after all-out exercise has far-reaching ramifications for athletes. The autonomic nervous system maintains physiological homeostasis during abrupt changes in physical states and is surmised to differ significantly between athletes and nonathletes. In general, it has been suggested that non-athletes have lower parasympathetic tone than athletes. Although this could not be substantiated from this study as data was obtained from high-performance athletes, athletes and non-athletes are nonetheless expected to demonstrate discrepant autonomic modulations after short maximal exercise.

One sprinter was excluded from the study due to mental and physical exhaustion after participating in a 3day collegiate track and field competition as the key player of his team. Resting autonomic tone of this athlete fell below the normal range and albeit pronounced subjective symptoms, the athlete failed to report his status prior to the beginning of the study. Moreover, continu- ous depression of parasympathetic activity was observed in this athlete. These results suggested that additional stress load on athletes suffering from overtraining syndrome could potentially prolong recovery. Despite reports of markedly higher vagal tone of athletes than nonathletes at rest, recovery after exercise also depends largely on this branch's activity. In principle, cardiac autonomic assessment using HRV is analogous to the estimation of varying velocity of a moving vehicle from the coordination of the accelerator and brake. In other words, HRV is akin to the changing speed of a vehicle, while the operation of accelerator and brake corresponds to sympathovagal control of the heart. Because athletes must rapidly modulate an acutely elevated heart rate during rest, increasing the vagal tone has important implications. Our results indicated that $\mathrm{LF} / \mathrm{HF}$ ratio, an index of sympathetic tone, following maximal high knee lifts declined, and that HF, an index of parasympathetic function, elevated 10 minutes after exercise. These results were consistent with a shift in proportion of neutrophils and lymphocytes in the differential leukocyte count, thus implying an association between autonomic functions and white blood cell count. In addition, these findings also undergirded the potential interactions between sympathetic activity and granulocytes, and parasympathetic activity and lymphocytes as cited by Abo [14]. With confirmed significant alterations in HRV parameters and differential leukocyte count in athletes following brief maximal exercise, quantitative evaluation of autonomic activity via HRV analysis and differential WBC emerge as plausible indices of conditioning level and physical capacity of athletes. The variance in neutrophil and lymphocyte ratios appears especially robust as an index, comparable to the assessment of autonomic functions.

Participants of this study involved first-class athletes who were anticipated to demonstrate superior autonomic modulation following exercise. In the case of non-athletes, however, post-exercise recovery was expected to be more sluggish. Since our test date coincided with the track and field season, recruitment of robust number of top Japanese athletes for the study was difficult. Several participants withdrew from the study due to fatigue from regular training. The sample population fell below the target size as great care was taken to prevent the physical burden of participating in the study from ethically interfering with competitions and training programs. Moreover, the nature and intensity of the exercise stress test were such that only the very best athletes in the country could perform them adequately, and the selection of such athletes with comparable athletic capacity resulted in a maximum sample size of five for this study. Despite the small sample, this is the first study to evaluate autonomic resilience in sprinters after maximal exercise, and 
therefore we believe that our results can serve as a basis for continued exploration of autonomic modulations in athletes. While different results may have been observed had athletes from various disciplines participated in the study, sprinters were specifically selected because of their training emphasis on basic exercises and resulting ability to demonstrate rapid changes in HRV.

According to our results, a greater sample size will minimize the individual differences in pre and post exercise parameters that were observed in this study. Additionally, future studies that provide a comparison with a control group and analyses of graded exercise intensity and post-exercise intervention will complement our study to deepen the understanding of the correlation between the autonomic nervous system and differential leukocyte count in athletes.

\section{REFERENCES}

[1] Hedelin, R., Wiklund, U., Bjerle, P., et al. (2000) Cardiac autonomic imbalance in an overtrained athlete. Medicine and Science in Sports and Exercise, 32(9), 1531-1533.

[2] Hayano, J., Sakakibara, Y., Yamada, A. and Mukai, S. (1991) Accuracy of assessment of cardiac vagal tone by heart rate variability in normal subjects. American Journal of Cardiology, 67(2), 199-204.

[3] Tanabe, S., Terao, T. and Nakano, S. (1993) Sympathovagal balance in young athletes. Tokai Journal of Sports Science and Medicine, 5, 44-49.

[4] Tanabe, S., Terao, T. and Nakano, S. (1995) Comparison of sympathovagal balance between the middle aged and young athletes. Tokai Journal of Sports Science and Medicine, 7, 75-82.

[5] Perini, R. and Veicsteinas, A. (2003) Heart rate variability and autonomic activity at rest and during exercise in various physiological conditions. European Journal of Applied Physiology, 90(3-4), 317-325.

[6] Sawada, Y. (1999) Heart rate variability, Is it available in psychophysiological research. Japanese Journal of Cancer Research, 26, 8-13.

[7] Yokoi, M. and Yamazaki, K. (1995) Cardiac autonomic nervous activity interaction under mental stress. A study using the corrected heart rate variability indexes. Tokai Journal of Sports Science and Medicine, 7, 75-82.

[8] Abo, T. (2002) Autonomic nervous regulation of leukocytes. Japanese Journal of Physical Fitness and Sports Medicine, 51, 10-11.

[9] Abo, T. (1999) Regulation of leukocytes by the autonomic nervous system, new rule on the cooperation of neuro-endocrine-immune systems (on the functions of the autonomic nervous system). Japanese Journal of Psychosimatic Medicine, 39, 67-74.

[10] Goto, K., Matuura, H. and Muramoto, K. (2002) Estimate of autonomic nervous system function by heart rate variability analysis [in Japanese] IEICE technical report. $M E$ and Bio Cybernetics, 102(507), 13-16.

[11] Hadase, M., Azuma, A. and Zen, K. (2004) Very low frequency power of heart rate variability is a powerful predictor of clinical prognosis in patients with congestive heart failure. Japanese Circulation Society, 68(4), 343347.

[12] Tanabe, S., Yoshioka, K. and Yamashita, Y. (1994) Sympathetic and parasympathetic activities at rest and after exercise in judo athletes. Tokai Journal of Sports Science and Medicine, 6, 35-42.

[13] Suzuki, K., Saoto, H. and Endo, T. (1995) Variation of neutrophil active oxygen and differential count of leukocyte's during maximal exercise load in athletes. Japanese Journal of Physical Fitness and Sports Medicine, 44(6), 747.

[14] Abo, T. (2002) Number and function of leukocytes are regulated by the autonomic nervous system. Journal of International Society of Life Information Science, 20(1), 171-189. 